\title{
Hereditary xanthinuria type II associated with mental delay, autism, cortical renal cysts, nephrocalcinosis, osteopenia, and hair and teeth defects
}

\author{
R Zannolli, V Micheli, M A Mazzei, P Sacco, P Piomboni, E Bruni, C Miracco, M M de Santi, \\ P Terrosi Vagnoli, L Volterrani, L Pellegrini, W Livi, B Lucani, S Gonnelli, A B Burlina, G Jacomelli, \\ F Macucci, L Pucci, M Fimiani, J A Swift, M Zappella, G Morgese
}

J Med Genet 2003;40:121 (http://www.jmedgenet.com/cgi/content/full/40/11/121)

$\mathrm{H}$ ereditary xanthinuria, a defect of purine metabolism, results from a genetic deficiency of xanthine oxidoreductase (XOR type I; OMIM \#278300), or both XOR and aldehyde oxidase deficiency (type II; OMIM \#603592). The human gene encoding for XOR is located at chromosome position $2 \mathrm{p} 22$, while that encoding aldehyde oxidase is at 2q33. ${ }^{1}$ The cause of type I hereditary xanthinuria clearly seems to be a mutation or mutations in the XOR gene. In type II deficiency, mutations in the genes coding for both XOR and aldehyde oxidase are unlikely, because these genes are located far apart, albeit both on chromosome $2 .{ }^{1}$ The human molybdenum cofactor sulphurase (HMCS) gene, encoding an enzyme for sulphuration of the desulpho molybdenum cofactor of XOR and aldehyde oxidase, has been found mutated in two independent patients with classical xanthinuria type II. $^{2}$ Both types of hereditary xanthinuria are clinically similar, but whereas patients with type I can metabolise allopurinol, those with type II cannot. ${ }^{1}$ Both types are characterised by plasma uric acid concentrations below $5 \mu \mathrm{mol} / \mathrm{l}$ and plasma xanthine concentrations over $10 \mu \mathrm{mol} / \mathrm{l}$. Urinary excretion of uric acid is low or undetectable, and that of xanthine is elevated. Less than half the affected people have symptoms, which are caused by deposition of xanthine in the urinary tract. This results in haematuria or renal colic, and rarely, in acute renal failure or chronic complications related to urolithiasis. Muscle pains caused by xanthine deposition occur in a minority of cases. ${ }^{1}$ To the best of our knowledge, associations of hereditary xanthinuria with mental delay and autism have never been described. We report here a case of a child with hereditary xanthinuria type II with mental delay and autism, cortical renal cysts, osteopenia, hair and teeth defects, and a range of behavioural symptoms.

\section{CASE REPORT}

The proband was an 11 year old boy (fig lA), who was referred to our clinic mainly for mental impairment, poor speech, and behavioural problems. He was the youngest of four siblings. The older siblings were described as being apparently normal. The child was born at term after an uneventful pregnancy, from apparently healthy non-consanguineous parents. Except for congenital hypertrichosis (mainly on the thigh, shoulder, and face), he apparently had no serious health problems. His physical growth was normal, but delayed psychomotor development and behavioural problems were apparent at an early stage. His karyotype was normal: $46 \mathrm{XY}$. At 11 years of age, his weight was $55 \mathrm{~kg}$ (>97th centile), his height was $148 \mathrm{~cm}$ (75th-90th centile), his body mass index was $25 \mathrm{~kg} / \mathrm{m}^{2}$ (>97th centile), and his head circumference was $54 \mathrm{~cm}$ (75th centile); Tanner

\section{Key points}

- We report here an 11 year old boy with type II hereditary xanthinuria (OMIM \#603592), mental delay, autistic features, vocal and motor tics, cortical and renal cysts, osteopenia, hair and teeth defects, and a range of behavioural symptoms, including aggressiveness with temper tantrums, and attention deficit hyperactivity disorder.

- To the best of our knowledge, these associations have not been described before.

stage P3-G2. He had no apparent dysmorphism. A macroscopic skin examination revealed congenital hypertrichosis as lanugo on his face, mainly on his upper lip, left eyebrow, and shoulder (images not shown). His teeth, nails, hair, and sudation were apparently normal. A skin biopsy, performed in an apparently normal area of the skin (left arm) proved normal. Despite the subject displaying apparently normal head hair, microscopy showed non-specific abnormalities in the shape of the shaft (fig 1B). Children's hairs normally have the gross form of an elliptical cylinder but approximately $2 \%$ may show slight longitudinal grooving of the surface at one of the extremities of the minor axial diameter (J A Swift, personal communication). The hairs of the proband, in contrast, exhibit a much higher than normal level of longitudinal grooving and localised concavities of their external surfaces. For whatever underlying reason, this must accord with an abnormally high incidence of peripheral distortion to the follicular apparatus of the hair. The absence of alternating bright and dark zones under polarising microscopy ruled out trichothiodystrophy (low sulphur content). Panoramic $x$ ray films (fig IC) showed hypodontia (tooth agenesis for $1.5,2.5)$, and microdontia $(2,4)$.

Mild autism was diagnosed according to the criteria for autistic disorders in the Diagnostic and Statistical Manual of Mental Disorders, 4 th ed. ${ }^{3}$ On the Autistic Behaviour Checklist, ${ }^{4}$ he scored 53, confirming autism of moderate degree. He had mental retardation and poor speech with an IQ (Wechsler Intelligence Scales for Children, Revised) of 41 . He had electroencephalogram (EEG) abnormalities and a range of behavioural symptoms, including aggressiveness with temper

Abbreviations: ADSL, adenylosuccinate lyase; $E E G$, electroencephalogram; HMCS, human molybdenum cofactor sulphurase; MRI, magnetic resonance imaging 
tantrums, attention deficit hyperactivity disorder, and vocal and motor tics. The EEG showed sporadic spikes in the right centrotemporal regions, and paroxysmal slowing in the frontal regions. A high field ( $1.5 \mathrm{~T}$ ) brain magnetic resonance imaging (MRI) scan showed mild right plagiocephaly (images not shown). There were no apparent areas of cortical dysplasia or periventricular heterotopia. Ophthalmological examination showed normal fundus oculi and visual evoked potentials. Physical and instrumental ear, nose, and throat examinations (tonal audiometric examination and impedentiometry) were normal. Echocardiography showed normal cardiac performance. Ultrasound examination of the genitourinary system revealed a group of several cortical cysts in the mesorenal region of his right kidney (diameter range 7$35 \mathrm{~mm}$, image not shown), and another single cortical cyst in the upper polar region (maximum diameter $8 \mathrm{~mm}$ ): a rare finding for a child of this age. ${ }^{5}$ There was a large pelvic stone in his left kidney (maximum diameter $2 \mathrm{~cm}$ ) and a second stone in an inferior calyx, causing distension of the intrarenal collecting system (images not shown). All the findings were also documented by conventional excretory intravenous urography. A radiological examination (plain abdominal $x$ ray film) showed the absence of radiopaque stones, excluding the presence of calcium deposits. High resolution computed tomography showed an increased volume of both kidneys with stones in the left side (fig lD), and several cysts and nephrocalcinosis in both sides (fig lE). Screening for metabolic disorders (high performance liquid chromatography, capillary electrophoresis linked techniques, or tandem mass spectroscopy) detected markedly decreased uric acid concentrations in the plasma $(0.66 \mu \mathrm{mol}$; normal range $148-$ $363 \mu \mathrm{mol})$ and urine $(0.012 \mathrm{mmol}$ per mmol creatinine; normal $1.5(0.3))$. There were grossly increased xanthine concentrations in the plasma $(40 \mu \mathrm{mol}$; normal range $0.3-$ 3.0 ) and urine $(0.1 \mathrm{mmol}$ per mmol creatinine; normal 0.03 $(0.01))$, and increased hypoxanthine concentration in $24 \mathrm{~h}$ urine (0.118 mmol per mmol creatinine; normal 0.03 (0.01)). Nucleotide patterns and purine nucleoside phosphorylase activity measured in erythrocytes were within the normal range, thus ruling out a congenital deficiency of this enzyme. Deficiencies of xanthine oxidoreductase (type I), or both XOR and aldehyde oxidase deficiency (type II), or deficiency of molybdenum cofactor (OMIM \#252150) were suspected. The lack of sulphite or sulphocysteine in his urine as measured by tandem mass spectroscopy, with normal levels of cysteine, ruled out sulphite oxidase or molybdenum cofactor deficiencies. To discriminate between types I and II xanthinuria, an allopurinol test was performed. Administration of $300 \mathrm{mg}$
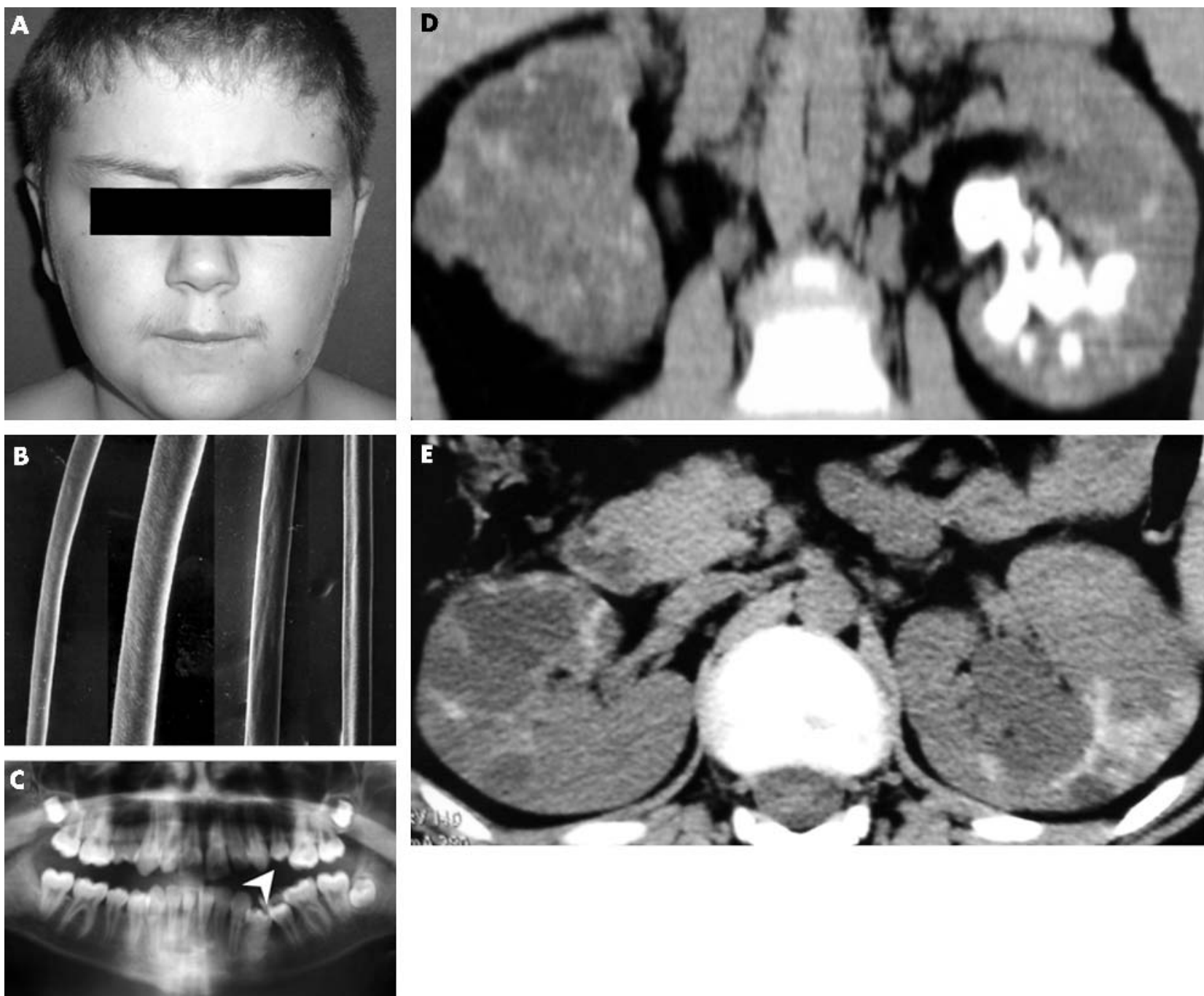

Figure 1 (A) Proband, an 11 year old child. (B) Scanning electron micrograph of a hair showing the irregular shape of the shaft. (C) Panoramic $x$ rays of the child's teeth showing agenesis for 1.5, 2.5, and microdontia (2.4, arrow). (D, E) High resolution computed tomography of the kidneys showing (D) stones and (E) cortical cysts and nephrocalcinosis. 
allopurinol was followed by blood collection after 90 min and urine collection for the following $24 \mathrm{~h} .{ }^{6}$ The absence of appreciable oxipurinol levels in plasma ${ }^{1}$ confirmed an inability to metabolise the drug via aldehyde oxidase, thus leading to the diagnosis of type II xanthinuria. For direct demonstration of deficient activity of XOR and aldehyde oxidase, a needle biopsy of the liver would have been necessary, because both enzymes are mainly expressed by this tissue, but this was not performed as it is seldom indicated for clinical purposes. ${ }^{1}$ All other routine biochemical tests and thyroid functions were normal. Osteopenia and phalangeal synostosis between the second and third bone of the fifth digit of the left foot showed up on $x$ ray film (image not shown). Calcium-phosphorus metabolism showed low values of $24 \mathrm{~h}$ calciuria ( $23 \mathrm{mg} / 24 \mathrm{~h}$; normal 100-300), low magnesuria ( $5 \mathrm{mg} / 24 \mathrm{~h}$; normal 73-121) and normal values of phosphaturia, calcitonin, parathyroid hormone, serum alkaline phosphatase, and serum bone alkaline phosphatase (bone ALP) measured with the Tandem (R) Ostase assay (Beckman Coulter Inc., Fullerton, CA, USA). ${ }^{7}$ Lumbar spine dual-energy $x$ ray absorptiometry showed clear osteopenia (Z score -2 ).

\section{DISCUSSION}

We report here an 11 year old boy with type II hereditary xanthinuria, mental delay, autistic features, vocal and motor tics, cortical renal cysts, osteopenia, hair and teeth defects, and a range of behavioural symptoms, including aggressiveness with temper tantrums, and attention deficit hyperactivity disorder. To the best of our knowledge, these associations have not been described previously.

Although the exact prevalence of metabolic abnormalities in autism spectrum disorders is unknown, several metabolic defects have been associated with autistic symptoms. These include phenylketonuria, histidinaemia, dihydropyrimidine dehydrogenase deficiency, and 5 '-nucleotidase superactivity. ${ }^{8}$ Patients affected by adenylosuccinate lyase (also called adenylosuccinase) deficiency (ADSL), a defect in the purine de novo synthesis pathway (OMIM \#103050), present with autistic features and normal level of uric acid. This enzymatic defect is associated with a pure encephalopathy comprising variable association of symptoms: psychomotor retardation, epilepsy, hypotonia (axial or generalised), peripheral hypertonia, and secondary feeding problems. ${ }^{9}$ Brain MRIs are normal or reveal myelin disturbances ranging from areas of increased T2 signal in white matter to severe leukodystrophy. ${ }^{9}$ The prognosis is poor for these patients. The symptoms of ADSL deficiency have been attributed to impaired synthesis of purine nucleotides or to toxic effects of the accumulating succinylpurines. Damage to brain functions could thus be caused by the interference of succinylpurines with glucose metabolism. ${ }^{10}$ Many patients with classic infantile autism exhibit uric acid excretion at more than two standard deviations above the normal mean. These hyperuricosuric autistic individuals may comprise approximately $20 \%$ of the autistic population. ${ }^{8}$ Although in this population a specific enzyme defect responsible for accelerated purine synthesis has not been identified, the abnormal ratio of adenine to guanine nucleotides suggest a defect in purine nucleotide interconversion. ${ }^{8}$ The clinical and biochemical picture of patients with hereditary type II xanthinuria seem quite different, notwithstanding the presence of mental delay and autistic disorders. The prognosis for our patient with hereditary type II xanthinuria is excellent, if his renal function can be conserved. ${ }^{1}$ Moreover, this child was not hypericosuric but hypouricaemic and hypouricosuric. He had no accumulation of succinylpurines, but instead demonstrated hyperxanthinaemia. Whether this accumulation of xanthine could be responsible for determining his autistic behaviour and mental impairment is unknown. Mental delay, but lack of autism, has also been described in individuals with molybdenum cofactor deficiency, another biochemical pattern showing hypouricaemia, hypouricuria and hyperxanthinuria with hyperxanthinaemia. In this defect, sulphide compound accumulation is present in the blood, because of sulphite oxidase deficiency. Unlike hereditary xanthinuria, this is a serious and fatal disease.

In addition to hereditary xanthinuria, mental delay and autistic features and a range of behaviour symptoms, it is noteworthy that our child presented with several other abnormalities: cortical renal cysts, nephrocalcinosis, osteopenia, hair and teeth defects. This suggests a novel defect of both ectodermal and mesodermal tissues.

Cysts have previously been described in individuals with ectodermal defect, ${ }^{11-13}$ mainly in the brain and cutis. Khoury et al. ${ }^{14}$ have described one individual with ED with renal cysts in the spongy medulla in association with congenital absence of teeth (anodontia). Cortical renal cysts are very rare in children, ${ }^{5}$ and to the best of our knowledge, have never been described in individuals with ED.

Nephrocalcinosis is another uncommon feature in childhood. The main causes are renal tubular acidosis, hyperparathyroidism, and medullary sponge kidney. It is also seen where there is hypercalcaemia or hypercalciuria from any aetiology. ${ }^{15}$ Nephrocalcinosis has been reported by Kessel et al. ${ }^{15}$ in an 18 month old infant with Jansen type metaphyseal chondrodysplasia, and in a neonate with McCune Albright syndrome who displayed atypical skeletal appearances and had multiple ovarian cysts. Nephrocalcinosis and renal cysts have been described by Moudgil et $a l^{16}$ in a child with apparent mineralocorticoid excess syndrome, suggesting that the development of nephrocalcinosis and renal cysts may be associated with chronic long-standing hypocalaemia. However, our case seems quite different. Osteopenia has previously been observed in children with ectodermal dysplasia. ${ }^{17-20}$

In conclusion, we report this further case of an ED syndrome associated with a wide ranging defect, in which specific ED findings were subtle and intriguing as in several other cases previously described. ${ }^{17-20}$ At present, it is not easy to hypothesise which type of gene/s could be involved in this novel and complex syndrome. Nevertheless, this further case makes stronger the idea that EDs are not a result of a general "ectodermal" abnormal defect, but more precisely, of systemic pathological conditions. ${ }^{21} 22$

Electronic Database Information: URLs for data in this article are as follows. Online Mendelian Inheritance in Man (OMIM): http:// www.ncbi.nlm.nih.gov/Omim/. Center for Medical Genetics, Johns Hopkins University, Baltimore, MD, and the National Center for Biotechnology Information, National Library of Medicine, Bethesda, MD: \#278300 xanthinuria, type I; \#603592 xanthinuria, type II; \#252150 molybdenum cofactor deficiency; \#103050 adenylosuccinate lyase (ADSL).

\footnotetext{
Authors' affiliations

R Zannolli, F Macucci, L Pucci, G Morgese, Department of Pediatrics, Obstetrics and Reproductive Medicine, Section of Pediatrics, Policlinico Le Scotte, University of Siena, Siena, Italy

V Micheli, G Jacomelli, Department of Molecular Biology, Section of Biological Chemistry, University of Siena, Siena, Italy

M A Mazzei, P Sacco, P Terrosi Vagnoli, Department of Radiology and Orthopaedics, Policlinico Le Scotte,University of Siena, Siena, Italy L Volterrani, Department of Human Pathology and Oncology, Section of Radiology and Radiotherapy, Policlinico Le Scotte, University of Siena, Siena, Italy

P Piomboni, E Bruni, Department of Pediatrics, Obstetrics and Reproductive Medicine, and Center for the Study of Germinal Cells, CNR Siena, Policlinico Le Scotte, University of Siena, Siena, Italy
} 
C Miracco, M M de Santi, Department of Human Pathology and Oncology, Section of Pathological Anatomy and Histology, Policlinico Le Scotte, University of Siena, Siena, Italy

L Pellegrini, W Livi, Department of ENT, Policlinico Le Scotte, University of Siena, Siena, Italy

B Lucani, S Gonnelli, Department of Internal Medicine, Policlinico Le Scotte, University of Siena, Siena, Italy

A B Burlina, Department of Pediatrics, University Hospital Padua, Italy M Fimiani, Department of Dermatology, University of Siena, Italy J A Swift, Department of Human Anatomy and Cell Biology, University of Liverpool, Liverpool, UK

M Zappella, Pediatrics Neuropsychiatric Unit, Azienda Ospedaliera Universitaria Senese, Policlinico Le Scotte, University of Siena, Siena, Italy

Correspondence to: Raffaella Zannolli, MD, Department of Pediatrics, Obstetrics and Reproductive Medicine, Section of Pediatrics, Policlinico Le Scotte, University of Siena, Siena, Italy; zannolli@unisi.it

\section{REFERENCES}

1 Raivio KO, Saksela M, Lapatto R. Xanthine oxidoreductase - Role in human pathophysiology and hereditary xanthinuria. In: Scriver CR, Beaudet AL, Sly WS, Valle D, eds. The metabolic and molecular basis of inherited disease New York: McGraw Hill, 2001:2653-62.

2 Ichida K, Matsumura T, Sakuma R, Hosoya T, Nishino T. Mutation of human molybdenum cofactor sulfurase gene is responsible for classical xanthinuria type II. Biochem Biophys Res Commun 2001;282:1194-200.

3 American Psychiatric Association. Diagnostic and statistical manual of mental disorders, 4th ed. Washington, DC: DSM-IV, 1994.

4 Krug DA, Arick JR, Almond PJ. Autism screening instrument for educational planning: background and development. In: Gillan J, ed. Autism: diagnosis, instruction, management and research. Austin: University of Texas, 1979:71-89

5 Mercado-Deane MG, Beeson JE, John SD. US of renal insufficiency in neonates. Radiographics 2002;22:1429-38.

6 Yamamoto T, Higashino K, Kono N, Kawachi M, Nanahoshi M, Takahashi S, Suda M, Hada T. Metabolism of pyrazinamide and allopurinol in hereditary xanthine oxidase deficiency. Clin Chim Acta 1989;180:169-75.

7 Kress BC, Mizrahi IA, Armour KW, Marcus R, Emkey RD, Santora AC 2nd. Use of bone alkaline phosphatase to monitor alendronate therapy in individual postmenopausal osteoporotic women. Clin Chem 1999;45:1009-17.

8 Page T, Coleman M. Purine metabolism abnormalities in a hyperuricosuric subclass of autism. Biochim Biophys Acta 2000;1500:291-6.
9 Van den Berghe G, Jaeken J. Adenylosuccinate lyase deficiency. In: Scriver CR, Beaudet AL, Sly WS, Valle D, eds. The metabolic and molecular basis of inherited disease. New York: McGraw Hill, 2001:2653-62.

10 De Volder AG, Jaeken J, Van den Berghe G, Bol A, Michel C, Cogneau M, Goffinet AM. Regional brain glucose utilization in adenylosuccinase-deficient patients measured by positron emission tomography. Pediatr Res $1988 ; 24: 238-42$

11 Sener RN. A patient with ectodermal dysplasia, Joubert's syndrome, and brain cysts. Comput Med Imaging Graph 1998;22:349-51.

12 Celebi JT, Tanzi EL, Yao YJ, Michael EJ, Peacocke M. Mutation report: identification of a germline mutation in keratin 17 in a family with pachyonychia congenita type 2. J Invest Dermatol 1999;1 13:848-50.

13 Zannolli R, Mostardini R, Pucci L, Sorrentino L, Biagioli M, Perotti R, Guarna M, Hadjistilianou T, Zerega G, Pierluigi M, Franco B, D'Ambrosio A, Morgese $G$. Corpus callosum agenesis, multiple cysts, skin defects, and subtle ocular abnormalities with a de novo mutation $[45, X X, \operatorname{der}(5),+(5 ; 14)$ (pter;q1 1.2)]. Am J Med Genet 2001;102:29-35.

14 Khoury Z, Brezis M, Mogle P. Familial medullary sponge kidney in association with congenital absence of teeth (anodontia). Nephron 1988;48:231-3.

15 Kessel D, Hall CM, Shaw DG. Two unusual cases of nephrocalcinosis in infancy. Pediatr Radiol 1992;22:470-1.

16 Moudgil A, Rodich G, Jordan SC, Kamil ES. Nephrocalcinosis and renal cysts associated with apparent mineralocorticoid excess syndrome. Pediatr Nephrol 2000;15:60-2

17 Zannolli R, Inchingolo G, Serracca L, Miracco C, De Santi MM, Malandrini A Biagioli M, Perotti R, Baldi C, Nuti D, Polito E, Gonnelli S. Ectodermal dysplasia syndrome with eyebrow alopecia, ptosis, strabismus, nystagmus, joint laxity, cerebellar ataxia, and osteopenia. Am J Med Genet 2002; 113:111-13

18 Zannolli R, Conversano E, Serracca L, Di Bartolo RM, Molinelli M, Galluzzi P, Mazzei MA, Terrosi-Vagnoli P, Miracco C, De Santi MM, Vatti G, Coviello G, Malandrini A, Gonnelli S, Alessandrini C, Fimiani M. Cortical periventricular heterotopia with ectodermal dysplasia. Am J Med Genet 2002;1 13:385-9.

19 Zannolli R, Pierluigi M, Pucci L, Lagrasta N, Gasparre O, Matera MR, Di Bartolo RM, Mazzei MA, Sacco P, Miracco C, de Santi MM, Aitiani $P$, Cavani S, Pellegrini L, Fimiani M, Alessandrini C, Galluzzi P, Livi W, Gonnelli S, Terrosi-Vagnoli P, Zappella M, Morgese G. 18q-syndrome and ectodermal dysplasia syndrome: Description of a child and his family. Am J Med Genet 2003;116:192-9.

20 Zannolli R, Macucci F, Di Bartolo RM, Serracca L, Miracco C, de Santi MM, Giannini F, Malandrini A, Galluzzi P, De Robertis S, Hadjistilianou T, Perotti R, Fimiani M, Doldo T, Giorgetti R, Cavani S, Pierluigi M. Novel CNS syndrome and ectodermal dysplasia. Am J Med Genet 2003;116:385-389.

21 Priolo M, Silengo M, Lerone M, Ravazzolo R. Ectodermal dysplasias: not only 'skin' deep. Clin Genet 2000;58:415-30.

22 Priolo $M$, Lagana $C$. Ectodermal dysplasias: a new clinical-genetic classification. J Med Genet 2001;38:579-85. 